\title{
Self-assembled island formation in heteroepitaxial growth
}

\author{
Albert-László Barabási ${ }^{a}$ \\ Department of Physics, University of Notre Dame, Notre Dame, IN 46556
}

(October 13, 2018)

\begin{abstract}
We investigate island formation during heteroepitaxial growth using an atomistic model that incorporates deposition, activated diffusion and stress relaxation. For high misfit the system naturally evolves into a state characterized by a narrow island size distribution. The simulations indicate the existence of a strain assisted kinetic mechanism responsible for the self-assembling process, involving enhanced detachment of atoms from the edge of large islands and biased adatom diffusion.
\end{abstract}

Typeset using REVTEX 
Heteroepitaxial growth of highly strained structures has gained interest lately as it offers the possibility to fabricate islands with very narrow size distribution without any substrate patterning process. Thanks to their small size, these islands, coined self-assembling quantum dots (SAQD), are candidates for one dimensional electron confinement [1]. SAQD formation has been observed for a wide range of material/substrate combinations, including InAs on GaAs [1 [6], InGaAs on GaAs [7,8], AlInAs on GaAlAs [9], GeSi on Si [10,11], InP on InGaP [12], GaSb on GaAs [13] and ZnSe on ZnMnSe [14], indicating the existence of a not yet understood common mechanism governing the self-assembling process.

In this paper we investigate the kinetics of island formation during heteroepitaxial growth using a one dimensional model [15] that includes all microscopic elements common to the materials for which SAQD formation has been observed, namely deposition, activated diffusion, and strain relaxation at every deposition and diffusion event. Depositing 2ML atoms with lattice constant $a_{f}^{0}$ on a substrate with lattice constant $a_{s}^{0}$, we find that sufficiently large misfit, $\epsilon \equiv\left(a_{f}^{0}-a_{s}^{0}\right) / a_{s}^{0}$, leads to self-assembled island formation in the system. In particular, $\epsilon=5 \%$ and $7.5 \%$ leads to a narrowly peaked island size distribution, in contrast with a wide distribution for $\epsilon=0 \%$ and $2.5 \%$. We discuss the kinetic mechanism responsible for the selfassembling process, and compare our results with experimental work on SAQD formation. Since the model does not contain material dependent features, the mechanism responsible for self-organization is expected to be generic, applicable to a wide class of materials.

Monte Carlo Method and Stress Relaxation - To include stress in the model, we consider that the atoms interact harmonically with their nearest and next nearest neighbors [16]. The elastic strain energies are recalculated after every deposition or diffusion event, using a checkerboard relaxation method. The relaxation starts at the last active atom, and propagates radially outward, until in a two particle wide shell all relative displacements are smaller than a preset parameter $\delta$. The relaxation is iteratively restarted from the same origin until even in the closest shell the displacement is smaller than $\delta$. Radial relaxation is more efficient for our problem than algorithms relaxing the entire system, since most changes in the displacement occur where a particle moves, and decrease fast with the distance from 
the source. Trial runs with different values of $\delta$ indicated that for $\delta \leq 10^{-3}$ the results were practically identical. In the simulations we used $\delta=10^{-4}$.

The surface particles are allowed to hop to neighboring lattice sites, with an SOS condition, disallowing up or down jumps larger than one atom high. The hopping probability for an atom is proportional to $\exp \left[-\left(n E_{n}+E_{0}-E_{s}\right) / k_{B} T\right]$, where $n$ is the number of neighboring atoms, $E_{n}$ is the bond energy and $E_{0}$ is the diffusion barrier for an isolated atom on a stress free substrate [17]. The strain energy is given by $E_{s}=(c / 2) \sum\left(a_{i}-a_{i}^{0}\right)^{2} /\left(a_{i}^{0}\right)^{2}$, where $a_{i}^{0}$ and $a_{i}$ are the bulk and stretched bond lengths, $i$ running over the occupied nearest and next-nearest neighbors. We use $c=44 \mathrm{eV}$ for the force constant, a typical value for many semiconductors $\left[18, E_{n}=0.3 \mathrm{eV}, E_{0}=0.4 \mathrm{eV}\right.$ and $\mathrm{T}=800 \mathrm{~K}$. The substrate consists of $N=50 \mathrm{ML}$ atoms with lattice constant $a_{s}^{0}=1$, on which we deposit with a constant flux 2ML atoms with lattice constant $a_{f}^{0}=a_{s}^{0}(1+\epsilon)$. The system size is $L=200$ [19]. We identify as island every mound with height larger than 1ML, and define the base size of the island, denoted by $s$, as the lateral size of the island measured in the second monolayer (to distinguish it from the wetting layer). The islands are coherently strained and dislocations are not allowed.

Numerical results - The most convincing evidence of the stress induced self-assembling process is provided by the island size distribution, shown in Fig. 11a. For $\epsilon=0 \%$ and $2.5 \%$ the distribution is wide, i.e. the system contains islands of all sizes, with a small peak around $s=20$. However, for $\epsilon=5 \%$ and $7.5 \%$ the distribution has a narrow peak centered at $s=6$ for $\epsilon=5 \%$ and $s=5$ for $\epsilon=7.5 \%$.

The parameter capturing the dynamics of self-assembly in the system is the relative width, $w_{s} / \bar{s}$, shown in Fig. [1a, where $w_{s}^{2} \equiv \bar{s}^{2}-\bar{s}^{2}$ is the width of the island size distribution and $\bar{s}$ is the average island size. An increasing $w_{s} / \bar{s}$ indicates unbounded growth of fluctuations, while a decreasing one is a signal of self-organization in the system. As Fig. 1la indicates, for $\epsilon=0 \%$ and $2.5 \% w_{s} / \bar{s}$ increases continuously with coverage, while for $\epsilon=5 \%$ and $7.5 \% w_{s} / \bar{s}$ increases only until it reaches a peak at some small coverage $\theta_{c}$, after which it decays. The peak signals the onset of self-organization: for $\theta>\theta_{c}$ we witness a continuous increase in 
the uniformity of the island size. The peak is at $\theta_{c}=0.66 \mathrm{ML}$ for $\epsilon=5 \%$, and $\theta_{c}=0.15 \mathrm{ML}$ for $\epsilon=7.5 \%$, indicating that the self-assembling process is more effective for larger misfit.

An experimentally often measured quantity is the island density, $\rho$. As Fig. 11b indicates, for the stress free system $\rho$ has a peak at $1.33 \mathrm{ML}$, after which it decreases. This behavior is characteristic for homoepitaxy [21]: for small coverages the dynamics is dominated by island nucleation. After a certain $\rho$ is reached the incoming atoms are captured by the existing islands, prohibiting further island nucleation, and stabilizing the island density. Continuing the deposition leads to island coalescence, that results in a drop of the island density. Fig. 1]b is consistent with this scenario for $\epsilon=0 \%$ and $\epsilon=2.5 \%$. However, we observe no such peak for $\epsilon=5 \%$ and $7.5 \%$, indicating continuous island nucleation, without significant coalescence [20].

Mechanism of self-organization - The main difference between the stress free and the stressed system comes in two strain related effects, that we discuss separately.

(a) Strain lowers the energy barrier for diffusion, thus making diffusive hops more probable. Fig. 2 shows the strain energy in the vicinity of an island for $\epsilon=7.5 \%$, indicating that the substrate is strained and that $E_{s}$ decreases as we move away from the edge of the island. This means that if atoms are deposited near the island, strain biases their otherwise random motion, generating a net surface current $\mathbf{j}=-\nabla \mu(x)$, where $\mu(x)$ is the local chemical potential [21]. The chemical potential is $\mu \simeq-\left(n E_{n}+E_{0}-E_{s}\right)$, where $n E_{n}+E_{s}$ is independent of the atom position for an isolated adatom on a flat surface. The only contribution to the current comes from the position dependence of the strain energy, leading to $\mathbf{j} \simeq-\nabla E_{s}$, that points towards the decreasing strain direction. Thus the strain field around an island generates a net current of adatoms away from the island.

(b) For large islands the strain energy, $E_{s}$, at the edge becomes comparable to the bonding energy of the edge atom, $n E_{n}+E_{0}$ (with $n=1$ ), enhancing its detachment, thus leading to a gradual dissolution of the island. Such mechanism favors a smaller average island size and leads to a narrower island size distribution, as observed by Ratsch et al. [22].

The simultaneous action of (a) and (b) leads to a kinetic mechanism stabilizing the 
island size: as islands grow, a strain field develops, that helps to dissolute the edge atoms (effect (b)) and "pushes" them away from the islands (effect (a)). Furthermore, the newly deposited atoms also diffuse away from the larger islands (effect (a)). These combined effects slow the growth rate of large islands and increase the adatom density away from them, thus enhancing the nucleation of new islands. The newly nucleated islands are small, and so is the strain field around them, thus they grow at a much faster rate than the older and larger one. This eventually leads to a narrow island size distribution in the system (Fig. 11a). The final island size is determined by a dynamical balance between the adatom density, the strain induced current away from the islands and the strain energy of the island, governing the detachment of the edge atoms. If we could monitor in real time the growth, we would witness a continuous nucleation of islands, such that small islands grow fast, and stop growing after they reach a certain size.

Discussion - The observed behavior compares favorably with the main features of the experimentally observed SAQD formation. First, TEM observations of the strained islands document the existence of the strain field in the substrate [7,8,23]. As Fig. 22 shows, such a field is reproduced by our relaxation method, and is responsible for the current $\mathbf{j}$ discussed in (a). Second, it is experimentally established that the increasing coverage contributes mainly to an increase in the island density, and much less to the further increase in the size of the existing islands [2], which is reproduced by the simulations (see Fig. 1]b). Third, experiments on GaInAs growth on GaAs substrate document an increasing width in the early stages of the deposition process, followed by a gradual decay for larger coverages [目, 团. This is similar to the behavior shown in Fig. [1a: $w / \bar{s}$ decreases only after a certain coverage $\theta_{c}$ has been reached. Indeed, for small coverages the islands are both small and distant, thus the strain induced biased diffusion (a) and atom detachment (b) are not yet relevant, and the island formation essentially follows a strain-free path. Only when $\theta$ approaches $\theta_{c}$ the discussed strain-induced mechanisms reverse the growth in the relative width. Finally, the simulations indicate ordering in the distances between the islands, as observed for high island densities in some investigations [6]. 
I have benefited from enlightening discussions with J.K. Furdyna, M. Krishnamurthy, N.N. Ledentsov, J.L. Merz, M.S. Miller, K. Newman, and W. Seifert. 


\section{REFERENCES}

a) Email address: alb@nd.edu

[1] For a recent review see W. Seifert, N. Carlsson, M-E. Pistol, L. Samuelson, and L.R. Wallenberg, J. Progr. Crystal Growth Charact. Mater. 33423 (1996); P.M. Petroff, and G. Medeiros-Ribeiro, MRS Bulletin 21 (No 4), 50 (1996).

[2] D. Leonard, K. Pond, and P.M. Petroff, Phys. Rev. B 50, 11687 (1994).

[3] M.S. Miller, S. Jeppesen, D. Hessman, B. Kowalski, I. Maximov, B. Junno, and L. Samuelson, Solid-State Electr. 40, 609 (1996).

[4] N.P. Kobayashi, T.R. Ramachandran, P.Chen, and A. Madhukar, Appl. Phys. Lett. 68, 3299 (1996).

[5] J.M. Moison, F. Houzay, F. Barthe, L. Leprince, E. André, and O. Vatel, Appl. Phys. Lett. 64, 196 (1994).

[6] S. Ruvimov, P. Werner, F. Hatami, K. Scheerschmidt, J. Heydenreich, U. Richter, N.N. Ledentsov, M. Grundmann, D. Bimberg, V.M. Ustinov, A. Yu. Egorov, P.S. Kop'ev, and Zh. I. Alferov, Phys. Rev. B 51, 14766 (1995).

[7] D. Leonard, M.Krishnamurthy, C.M. Reaves, S.P. Denbaars, and P.M. Petroff, Appl. Phys. Lett. 63, 3203 (1993).

[8] A. Madhukar, Q. Xie, P. Chen, and A. Konkar, Appl. Phys. Lett. 64, 2727 (1994).

[9] R. Leon, S. Fafard, D. Leonard, J.L. Merz, and P.M. Petroff, Appl. Phys. Lett. 67, 521 (1995).

[10] R. Apetz, L. Vescan, A. Hartman, C. Diecker, and H. Luth, Appl. Phys. Lett. 66, 445 (1995); P. Schittenhelm, M. Gail, J. Brunner, J.H. Nutzel, and G. Abstreiter, ibid. 66 1292 (1995); M.Krishnamurthy, J.S. Drucker, and J.A. Venables, J. Appl. Phys. 69, 6461 (1991); D.E. Jesson, K.M. Chen, and S.J. Pennycook, MRS Bulletin 21 (No 4), 
$31(1996)$.

[11] G. Abstreiter, P. Schittenhelm, C. Engel, E. Silveira, A. Zrenner, D. Meertens, and W. Jager, Semic. Sci. Techn. (in press).

[12] N. Carlsson, W. Seifert, E. Peterson, M. Castillo, M.E. Pistol, and L. Samuelson, Appl. Phys. Lett. 65, 3093 (1994).

[13] F. Hatami, N.N. Ledentsov, M. Grundmann, J. Bohrer, F. Heinrichsdorff, M. Beer, D. Bimberg, S.S. Ruminov, P.Werner, U. Gosele, J. Heydenreich, U. Richter, S.V. Ivanov, B. Ya. Meltser, P.S. Kop'ev, and Zh. I. Alferov, Appl. Phys. Lett. 67, 656 (1995).

[14] S.H. Xin, P.D. Wang, A. Yin, M. Dobrowolska, J.L. Merz, and J.K. Furdyna, Appl. Phys. Lett. 69, 3884 (1997).

[15] The fact that the model is one dimensional is not expected to affect the nature of the self-organization process, but it can modify such quantities as the island size and critical coverage.

[16] B.G. Orr, D. Kessler, C.W. Snyder, and L.M. Sander, Europhys. Lett. 19, 33 (1992).

[17] Note that compressive (tensile) strain lowers (incerases) the energy barrier for diffusion [see M. Schroeder and D.E. Wolf (preprint)]. For SAQD formation the strain is compressive, thus we use a negative sign for the strain energy. This sign also determines the direction of the surface current near an island discussed under (a).

[18] W.A. Harrison, Electronic Structure and the Properties of Solids (Freeman, San Francisco, 1980).

[19] The time consuming strain relaxation limits the diffusion length, $\ell_{d}$. The typical $\ell_{d}$ in our simulations varies between 19 lattice sites for $\epsilon=0 \%$ to 31 for $\epsilon=7.5 \%$. This is much shorter than the experimental diffusion length, which is of order of hundreds of $\mathrm{nm}$. This decreases the average distance between the islands and their average size, since 
there will be a smaller surface area from where the islands can collect atoms. Physically, the curtained $\ell_{d}$ corresponds to growth with high flux.

[20] Note that in Fig. 1]b $\rho$ increases much faster for $\epsilon=7.5 \%$ than for $\epsilon=5 \%$. While for $\epsilon=5 \%$ a wetting layer is formed, for $\epsilon=7.5 \%$ all deposited atoms aggregate in islands. Thus we suspect that for $\epsilon=7.5 \%$ the strain energy is large enough to lead to the Volmer-Weber growth mode (3D island formation), in contrast with $\epsilon \leq 5 \%$, that grows in the Stranski-Krastanov growth mode. The detailed account of this transition will be presented elsewhere.

[21] For a recent review see A.-L. Barabási and H. E. Stanley, Fractal Concepts in Surface Growth (Cambridge University Press, Cambridge, 1995).

[22] C. Ratsch, A. Zangwill, and P. Smilauer, Surf. Sci. 314, L937 (1994).

[23] A. Ponchet, A. Le Corre, H. L'Haridon, B. Lambert, and S. Salaün, Appl. Phys. Lett. 67, 1850 (1995). 


\section{FIGURES}

FIG. 1. (a) Island size distribution measured after the deposition of 2ML atoms. Inset: Relative width $w_{s} / \bar{s}$ as a function of coverage. (b) Island density as a function of coverage. In all figures the symbols correspond to misfit values $0 \%(\circ), 2.5 \%(\square), 5 \%(\diamond)$, and $7.5 \%(\triangle)$.

FIG. 2. The strain energy around a typical island. The substrate (filled $\square$ ) and the islands on top of it (empty $\square$ ) are shown on the upper part of the figure. $E_{s}$ is the strain energy of an atom placed on the top of the substrate or on the island. For example, $E_{s}$ at $x=18$ is the strain energy

felt by the adatom shown by the circle on the top of the substrate. One can see that $E_{s}$ is the largest when the atom is at the edge of the island $(x=21,30) . E_{s}$ decays as the adatom moves away from the island, generating a net current, $\mathbf{j}(x)$, shown by the arrows. Note that $E_{s}$ does not decay to zero, since the monomer can locally stretch the substrate. 


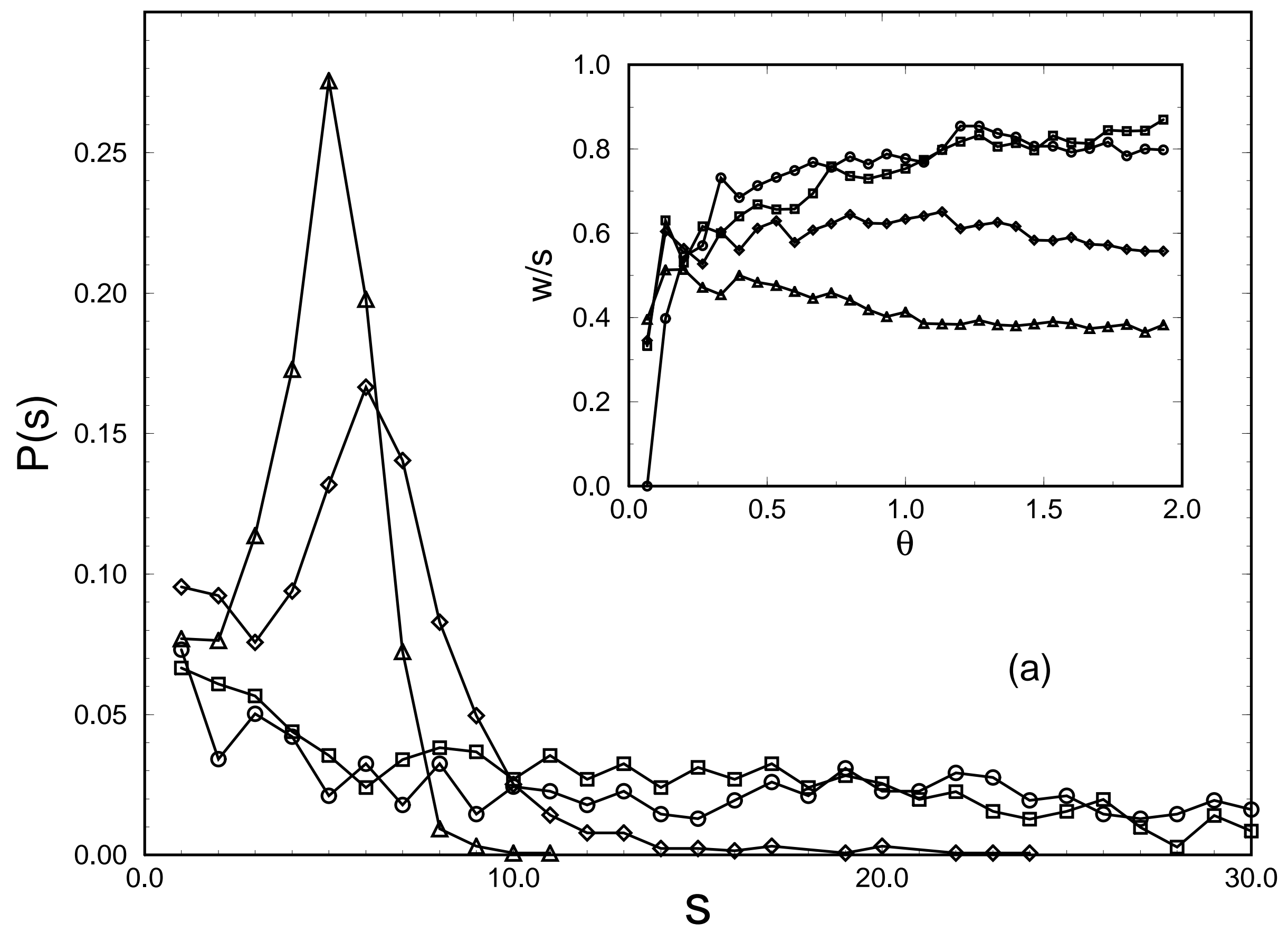




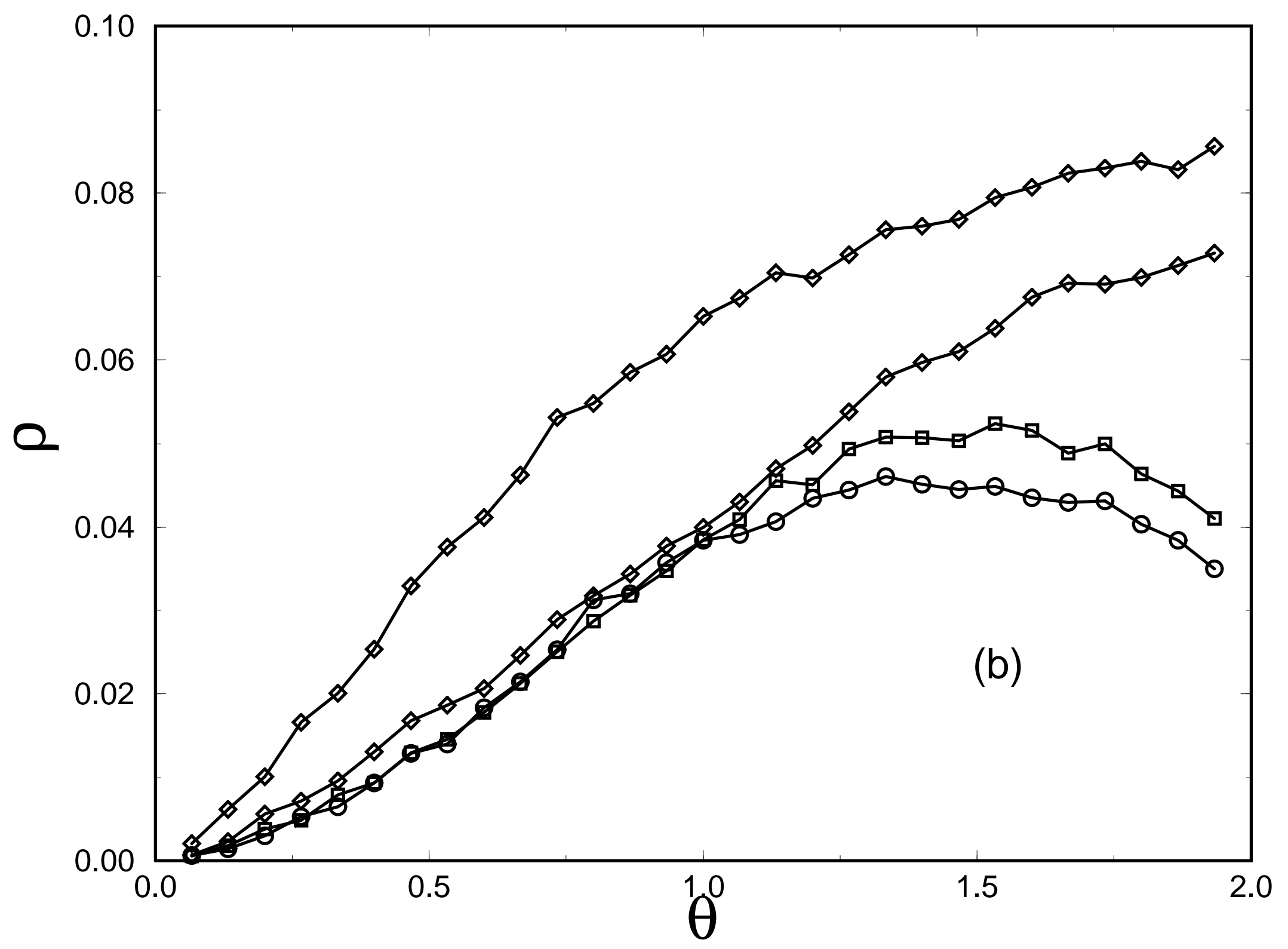




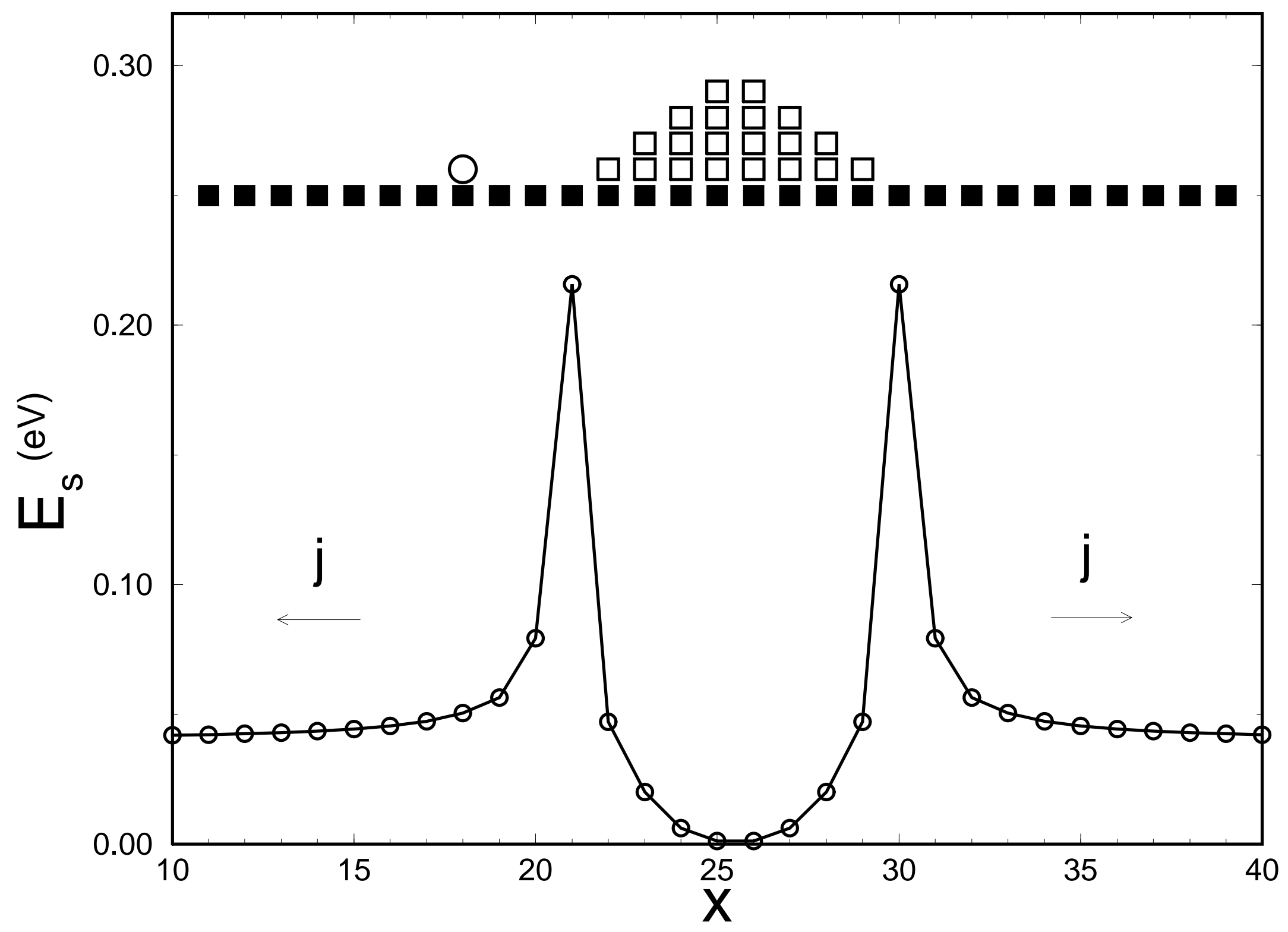

